\title{
Antiferromagnetic behavior in $\mathbf{S}=\mathbf{2}$ layered $\mathrm{RbMnF}_{4}$
}

\author{
M. C. Morón and F. Palacio \\ Instituto de Ciencia de Materiales de Aragón, CSIC-Universidad de Zaragoza, E-50009 Zaragoza, Spain
}

J. Rodriguez-Carvajal

Institut Laue-Langevin, BP 156X, F-38042 Grenoble, France

The magnetic properties of layered-perovskite $\mathrm{RbMnF}_{4}$ have been investigated by neutron powder diffraction. This compound exhibits a magnetic ordering below $T_{c}=3.7 \pm 0.2 \mathrm{~K}$. The resolution of the magnetic structure at $1.5 \mathrm{~K}$ indicates that $\mathrm{RbMnF}_{4}$ orders as a collinear antiferromagnet. The propagation vector is $\mathbf{k}=0$ and the magnetic moment of each sublattice has a value of $2.97(3) \mu_{B}$. The strong reduction of the observed magnetic moment should be attributed to a particularly high two-dimensional character of this compound.

\section{INTRODUCTION}

Fluorinated $\mathrm{Mn}^{3+}$ compounds provide a number of low-dimensional materials with potentially interesting structural and magnetic properties. ${ }^{1-3}$ The compounds of general formula $\mathrm{AMnF}_{4}$, where $\mathrm{A}$ refers to an alkali ion, tend to form layers of corner-sharing $\left[\mathrm{MnF}_{2} \mathrm{~F}_{4 / 2}\right]^{-}$octahedra separated by the alkali ions, their structure being of the $\mathrm{TlAlF}_{4}$ type. The octahedra are distorted due to both steric and Jahn-Teller effects. A direct consequence of this distortion is the increase of the single ion anisotropy of the $\mathrm{Mn}^{3+}$ ion. ${ }^{4-6}$

The size of the alkali ion also influences the magnetic properties of this family of layercd compounds. Thus, $\mathrm{NaMnF}_{4}$ and $\mathrm{KMnF}_{4}$ order as noncollinear antiferromagnets below, respectively, $13 \mathrm{~K}^{5}$ and $5.2 \mathrm{~K}$, ${ }^{6}$ while $\mathrm{CsMnF}_{4}$ has been described as a ferromagnet below $18.9 \mathrm{~K}^{7}$ Moreover, susceptibility measurements in powder samples of $\mathrm{RbMnF}_{4}$ indicates that this compound orders as an antiferromagnet below $T_{c}<4.5 \mathrm{~K}$. ${ }^{8}$ Such a diversity in the magnetic behavior opens interesting questions concerning the influence of the alkali ion and the relationship between crystal structure and magnetic properties in these compounds.

As part of our current investigation of the magnetostructural correlations within the $\mathrm{AMnF}_{4}$ series, we report in this work the magnetic behavior of $\mathrm{RbMnF}_{4}$ studied by neutron powder diffraction.

\section{EXPERIMENT}

$\mathrm{RbMnF}_{4}$ was prepared by controlled dehydration of $\mathrm{RbMnF}_{4} \cdot \mathrm{H}_{2} \mathrm{O}$ at $120^{\circ} \mathrm{C}$. The hydrated compounds were synthesized following already described methods. ${ }^{2,9}$

The neutron scattering experiments were carried out on the high-flux medium-resolution D1B $(\lambda \approx 2.52 \AA)$ powder diffractometer at the High Flux Reactor of the Institut Laue-Langevin in Grenoble (France). Diffraction patterns were recorded in the angular range $8^{\circ} \leqslant 2 \theta \leqslant 88^{\circ}$ between 1.5 and $4.8 \mathrm{~K}$.

The data were analyzed by using the program FULLPROF ${ }^{10}$ which allows the Rietveld refinement of multiphase patterns combining nuclear and magnetic structures.

\section{RESULTS AND DISCUSSION}

Neutron powder diffractograms taken in $\mathrm{RbMnF}_{4}$ as a function of the temperature are shown in Fig. 1. The enhancement observed in some of the Bragg reflections corresponds to the magnetic ordering of the sample. The thermal evolution of the magnetic reflections with the temperature in the range $1.5-4.8 \mathrm{~K}$ indicates that $\mathrm{RbMnF}_{4}$ exhibits magnetic ordering below the critical temperature $T_{c}=3.7 \pm 0.2 \mathrm{~K}$. To get a deeper insight into the nature of the magnetic couplings we have determined the magnetic structure of $\mathrm{RbMnF}_{4}$ at $1.5 \mathrm{~K}$.

In order to solve the magnetic structure of a given compound an accurate knowledge of the corresponding crystal structure in the ordered phase is required. The crystal structure of $\mathrm{RbMnF}_{4}$ belongs to the layered-perovskite structure. Therefore, the spatial arrangement of the atoms consists of layers of $\left[\mathrm{MnF}_{2} \mathrm{~F}_{4 / 2}\right]^{-}$corner-sharing octahedra separated by the $\mathbf{R b}$ atoms (Fig. 2). The octahedra are both tilted and distorted due to the double influence of the Jahn-Teller distortion and the steric effect of the alkali ion. There are three different $\mathrm{Mn}-\mathrm{F}$ distances in the octahedra, the shortest corresponding to the axial fluorine atoms, the long axis of the octahedra being antiferrodistortively or-

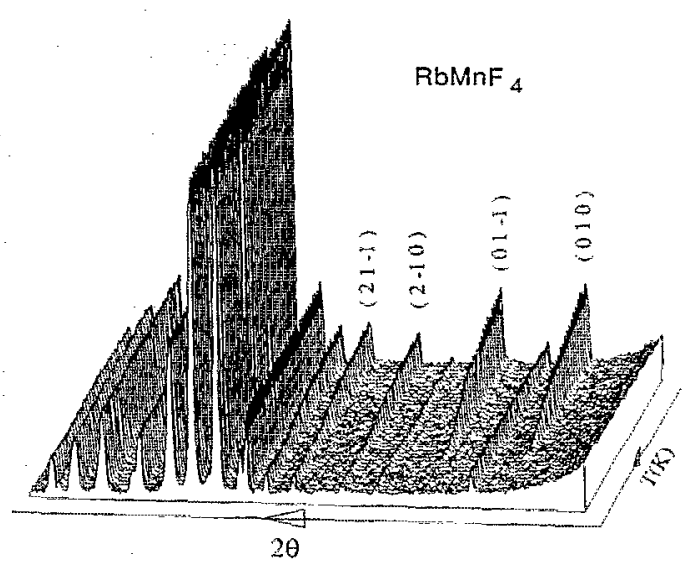

FIG. 1. 3d plot of the neutron powder diffraction patterns as a function of the temperature in the range $8^{\circ} \leqslant 2 \theta \leqslant 88^{\circ}$ for $\mathrm{RbMnF}_{4}(\Delta T=1.5-4.8$ K). The index of the Bragg peaks showing strong magnetic contribution are depicted. 


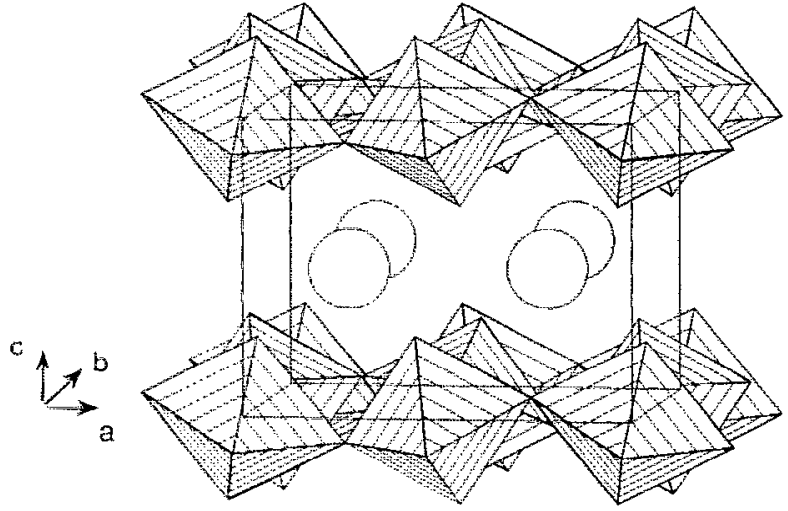

FIG. 2. View of the unit cell of $\mathrm{RbMnF}_{4}$ showing the layered character of this compound. Open circles represent the $\mathrm{Rb}$ atoms and octahedra the $\left[\mathrm{MnF}_{2} \mathrm{~F}_{42}\right]^{-}$units.

dered within the layers. $\mathrm{RbMnF}_{4}$ crystallizes in the space group $P_{1} / a$, with $a=7.8119(4) \AA, b=7.7761(4) \AA$, $c=6.0469(3) \AA, \beta=90.443(4)^{\circ}$ and $Z=4$ at room temperature, being isomorphic to $\mathrm{KMnF}_{4}$. The $c$ axis has been taken perpendicular to the $\mathrm{MnF}_{4}$ layers. No structural phase transition has been detected down to $1.5 \mathrm{~K}$. In order to determine the magnetic structure of $\mathrm{RbMnF}_{4}$ it is also important to consider that there are two independent $\mathrm{Mn}$ sites ( $a$ and $c$ ). Moreover, the magnetic atoms are located at a symmetry point $\overline{1}^{6}{ }^{6}$

Taking into account the symmetry information, the magnetic structure was solved by trial and error. Due to the fact that all the magnetic reflections can be indexed in the crystallographic unit cell, the propagation vector of the magnetic structure is $\mathbf{k}=\mathbf{0}$. Since the $\mathbf{M n}$ atoms are in special positions, the magnetic structure factor can be easily written and a rapid test, as a function of the Miller indices of the most intense magnetic reflections, can be

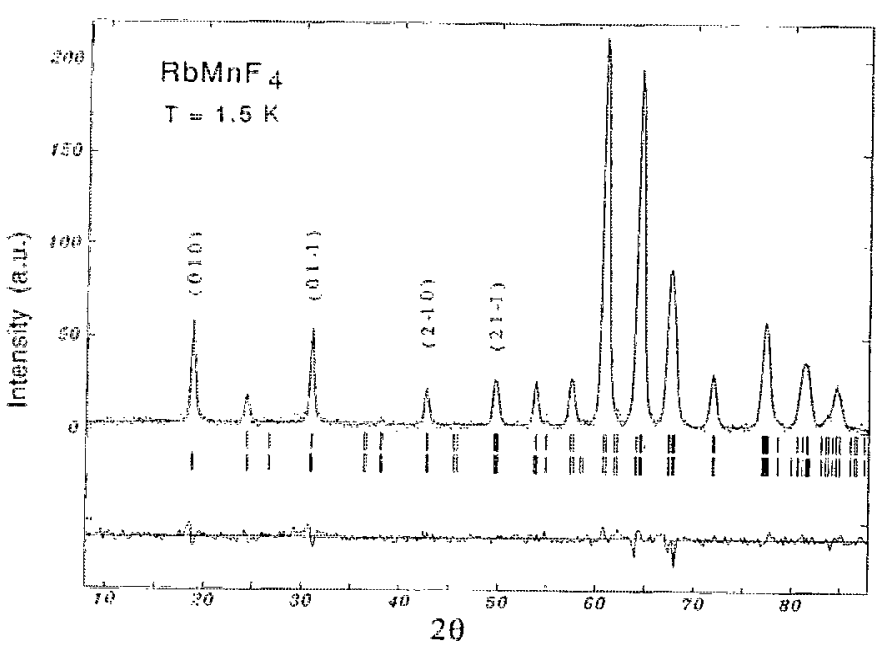

FIG. 3. Observed (dotted eurve) and calculated (full curve) nuclear and magnetic intensities of $\mathrm{RbMnF}_{4}$ at $1.5 \mathrm{~K}$. The difference pattern $Y_{\text {obs }}$ $-Y_{\text {cali }}^{*}$ is given at the bottom of the figure on the same scale. Angular positions of the allowed Bragg reflections are indicated by small bars.
TABLE I. Magnetic moment components, in Bohr magnetons, along the three crystallographic axes for $\mathrm{RbMnF}_{4}$ at $T=1.5 \mathrm{~K}(\mathrm{k}=0), \mathrm{Mn}(a)$ and $\mathrm{Mn}(c)$ represent the two independent $\mathrm{Mn}$ sites.

\begin{tabular}{cccccccc}
\hline & $x$ & $y$ & $z$ & $m_{x}$ & $m_{y}$ & $m_{z}$ & $m\left(\mu_{B}\right)$ \\
\hline $\operatorname{Mn}(a) 1$ & 0 & 0 & 0 & $1.97(4)$ & $1.97(4)$ & $1.0(1)$ & $2.97(3)$ \\
$\operatorname{Mn}(a) 2$ & $1 / 2$ & $1 / 2$ & 0 & - & - & - & \\
$\operatorname{Mn}(c) 1$ & $1 / 2$ & 0 & 0 & + & + & + & \\
$\operatorname{Mn}(c) 2$ & 0 & $1 / 2$ & 0 & - & - & - & \\
\hline
\end{tabular}

performed by hand. This permits the selection of the most probable magnetic structures.

The fit of our experimental data to the different models by using the Rietveld method implemented in FULLPROF shows a collinear antiferromagnetic structure for $\mathrm{RbMnF}_{4}$. The reliability factor $R_{M}$ is 7.0. The observed and calculated patterns at $1.5 \mathrm{~K}$ are presented in Fig. 3 while the projection of the magnetic moment of each sublattice along the three crystallographic axes is shown in Table I. A [001] view of the crystal and magnetic unit cell of $R b M n F_{4}$ is shown in Fig. 4. The magnetic moment of each sublattice is 2.97 (3) $\mu_{B}$ at $1.5 \mathrm{~K}$. The expected value of the magnetic moment for a $\mathrm{Mn}^{3+}$ ion with $S=2$ is $4 \mu_{B}$; the strong reduction of the observed magnetic moment should be attributed to a particularly high two-dimensional character of this compound, as reported for $\mathrm{KMnF}_{4}{ }^{6}{ }^{6}$ The zero-point spin deviation of $2 \mathrm{~d}$ antiferromagnets due to quantum fluctuations is much larger than in $3 \mathrm{~d}$ antiferromagnets. ${ }^{11}$

Isotropic superexchange interaction does not impose any particular direction of the magnetic moments with respect to the crystal frame. Except in cases of topological frustration, or competition between nearest and nextnearest neighbor interactions, the pure isotropic exchange interaction tends just to keep the spins exactly parallel or antiparallel depending on its sign. Since the magnetic moments in $\mathrm{RbMnF}_{4}$ are collinear, anisotropic terms are not required to explain the magnetic structure of this compound. However, the noncollinear antiferromagnetic structures reported for $\mathrm{NaMnF}_{4}$ (Ref. 5) and $\mathrm{KMnF}_{4}$ (Ref. 6)

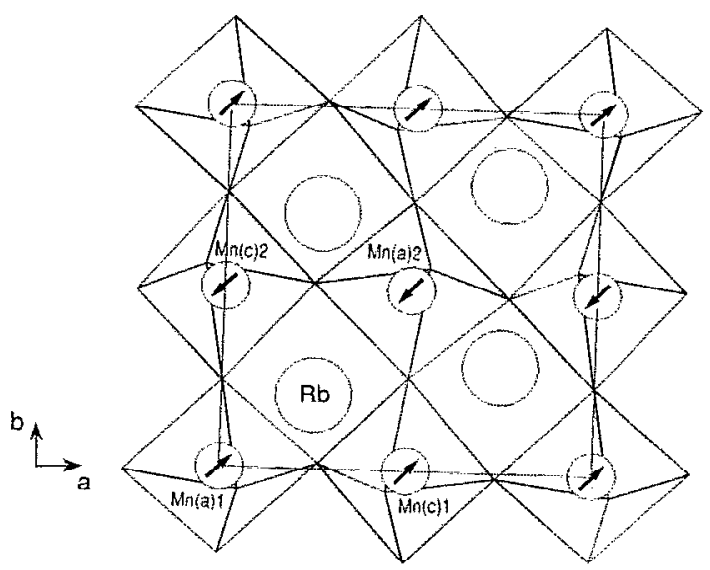

FIG. 4, [001] view of the crystal and magnetic unit cell of $\mathbf{R b M n F}_{4}$ showing the collinear orientation of the magnetic moments. The numbering of the magnetic atoms refers to Table $I$. 
indicate that anisotropic terms should have a nonnegligible contribution to the general exchange Hamiltonian describing the magnetic interactions in these two compounds. Moreover, the sign of the isotropic exchange interaction between nearest neighbors in $\mathrm{RbMnF}_{4}$ is positive (ferromagnetic) along the $a$ axis but negative (antiferromagnetic) along the $b$ axis (Fig. 4). Interestingly enough, the superexchange angle Mn-F-Mn along the $a$ axis is larger than along the $b$ axis. A more complete study about the relationship between the sign of the isotropic exchange interaction and the value of the superexchange angle within the $\mathrm{AMnF}_{4}$ series, is in progress.

\section{ACKNOWLEDGMENTS}

The research in Zaragoza has been supported by Grant No. MAT/91-681, from the Comision Interministerial de Ciencia y Tecnología.
'J. Pebler, W. Massa, H. Lass, and B. Ziegler, J. Solid State Chem. 71, 87 (1987).

${ }^{2}$ F. Palacio, M. Andrés, C. Esteban-Calderón, M. Martínez-Ripoll, and S. Garcia-Blanco, J. Solid State Chem. 76, 33 (1988).

${ }^{3}$ F. Palacio, M. Andrés, J. Rodriguez-Carvajal, and J. Pannetier, J. Phys: Condensed Matter 3, 2379 (1991).

${ }^{4}$ K. H. Wandner and R. Hoppe, Z. Anorg. Allg. Chem. 546, 113 (1987).

${ }^{5}$ M. Molinier, W. Massa, S. Khairoun, A. Tressaud, and J. L. Soubeyroux, Z. Naturforsch. B 46, 1669 (1991).

${ }^{6}$ M. C. Morón, F. Palacio, and J. Rodríguez-Carvajal (to be published).

${ }^{7}$ W. Massa and M. Steiner, J. Solid State Chem. 32, 137 (1980).

${ }^{8}$ P. Köhler, W. Massa, D. Reinen, B. Hofmann, and R. Hoppe, Z. Anorg. Allg. Chem. 446, 131 (1978).

${ }^{9} \mathrm{~V}$. Kaucic and P. Bukovec, J. Chem. Soc. (Dalton Trans.) 1512 (1979).

${ }^{10}$ J. Rodríguez-Carvajal, in Satellite Meeting on Powder Diffraction, abstracts of the XVth Conference of the International Union of Crystallography (Toulouse, 1990), p. 127; J. Rodriguez-Carvajal (unpublished).

${ }^{11}$ M. E. Lines, Phys. Rev. 164, 736 (1967). 